\title{
Shikonin induces apoptosis and G0/G1 phase arrest of gallbladder cancer cells via the JNK signaling pathway
}

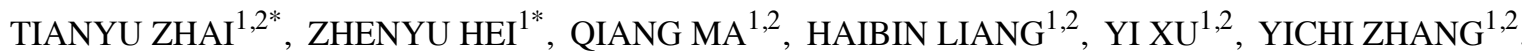 \\ LONGYANG JIN $^{1}$, CHAO HAN ${ }^{1}$ and JIANDONG WANG ${ }^{1,2}$ \\ ${ }^{1}$ Department of General Surgery and Laboratory of General Surgery, Xinhua Hospital Affiliated to \\ Shanghai Jiao Tong University School of Medicine; ${ }^{2}$ Institute of Biliary Tract Disease, \\ Shanghai Jiao Tong University School of Medicine, Shanghai 200092, P.R. China
}

Received April 21, 2017; Accepted September 29, 2017

DOI: $10.3892 /$ or.2017.6038

\begin{abstract}
Shikonin, a natural product isolated from the roots of Lithospermum erythrorhizon, is considered to have antitumor effects. Gallbladder cancer (GBC) is a prevalent biliary tract malignancy with few curative therapeutic stragegies and poor prognosis. In the present study, we detected the effects of shikonin on GBC cells as well as the underlying molecular mechanisms. The results demonstrated that GBC cell proliferation was inhibited by shikonin as determined by MTT and colony formation assays. Flow cytometry results demonstrated that shikonin treatment enhanced apoptosis and promoted G0/G1 phase arrest in the GBC cells. Western blot assay showed that shikonin induced mitochondrial-dependent apoptosis via the JNK signaling pathway. Moreover, shikonin suppressed tumor growth in mice bearing GBC-derived xenografts in a dose-dependent manner without side-effects. These results revealed that shikonin exhibits anticancer effects on GBC cells by inducing apoptosis and regulating the cell cycle. Taken together, shikonin may be a novel and safe chemotherapeutic agent for the treatment of GBC.
\end{abstract}

\section{Introduction}

Gallbladder cancer (GBC) is a prevalent biliary tract malignancy $(1,2)$, and the seventh most common gastrointestinal cancer worldwide $(3,4)$. The prognosis for GBC is extremely poor $(5,6)$, and GBC is usually diagnosed at a late stage due to the lack of specific symptoms $(7,8)$. The only curative therapy for GBC is surgical resection (9). The American College of

Correspondence to: Professor Jiandong Wang, Department of General Surgery, Xinhua Hospital Affiliated to Shanghai Jiao Tong University School of Medicine, 1665 Kongjiang Road, Shanghai 200092, P.R. China

E-mail:wjddoctor@sina.com

*Contributed equally

Key words: shikonin, gallbladder cancer, apoptosis, cell cycle arrest, in vivo
Surgeons reported in 2010 that the 5-year survival rate for stage IV GBC is $\sim 4 \%$ (10), resulting from early infiltration by lymphatic, perineural and hematogenous routes, as well as direct invasion into the liver (11). Thus, the identification of novel and promising agents for the treatment of $\mathrm{GBC}$ is urgently needed.

Shikonin (Fig. 1) is a compound isolated from the roots of Lithospermum erythrorhizon, a traditional medicinal plant (12). Numerous studies have demonstrated that shikonin exhibits antitumor effects such as inhibition of cancer cell proliferation (13), induction of apoptosis (14), attenuation of invasion and migration (15), and inhibition of proteasome activity (16), angiogenesis (17) and cancer cell glycolysis (18). However, no studies have been conducted concerning the effects of shikonin on GBC cells and the potential mechanisms involved. Therefore, the present study was designed to investigate the effect of shikonin on GBC cells in vitro and xenograft tumors in vivo, and explore the underlying molecular mechanisms underlying the effects. The present study may offer a promising agent for the treatment of GBC.

\section{Materials and methods}

Chemicals and reagents. Shikonin was obtained from the Shanghai Jinsui Biotechnology Co., Ltd. (Shanghai, China). After being dissolved in dimethyl sulfoxide (DMSO) at a stock solution $(0.05 \mathrm{~mol} / \mathrm{l})$, shikonin was stored at $-20^{\circ} \mathrm{C}$. Subsequent dilutions were conducted with culture medium. An equal proportion of vehicle was added to the control cells.

3-[4,5-Dimethylthiazol-2-yl]-2,5-diphenyl-tetrazolium bromide (MTT) and Hoechst 33342 were obtained from Sigma Chemical Company (St. Louis, MO, USA). An Annexin V/propidium iodide (PI) apoptosis kit was obtained from Invitrogen (Carlsbad, CA, USA). Primary antibodies against Bcl-2, Bax, cleaved caspase-9, cleaved caspase-3, cleaved PARP, cyclin D1, CDK4, JNK, phosphorylated JNK (p-JNK), GAPDH and secondary antibodies (goat anti-rabbit) were obtained from Cell Signaling Technology (Danvers, MA, USA).

Cell lines and culture. NOZ and EHGB-1 (human GBC) cell lines were obtained from the Shanghai Institute of Cell Biology, Chinese Academy of Sciences and grown in high-glucose 
Dulbecco's modified Eagle's medium (Gibco, Grand Island, NY, USA). The media contained $100 \mathrm{U} / \mathrm{ml}$ penicillin (HyClone, Logan, UT, USA), and 10\% fetal bovine serum (FBS; Gibco) and $100 \mu \mathrm{g} / \mathrm{ml}$ streptomycin. The cells were grown at $37^{\circ} \mathrm{C}$ in an atmosphere with $5 \% \mathrm{CO}_{2}$.

Cell viability assay. Cell viability was detected by the MTT assay. NOZ and EHGB-1 cells (2x10 $/$ well) were seeded into 96-well plates and incubated overnight. Then, the cells were treated with shikonin at various concentrations of $0,0.5,1,2$, 3 and $4 \mu \mathrm{mol} / \mathrm{l}$ for 24,48 and $72 \mathrm{~h}$. After treatment, $10 \mu \mathrm{l}$ of MTT solution $(5 \mathrm{mg} / \mathrm{ml})$ was added to each well and incubation was carried out at $37^{\circ} \mathrm{C}$ for $4 \mathrm{~h}$. The culture medium was then replaced with $100 \mu \mathrm{l}$ of DMSO. Absorbance of the solution at $490 \mathrm{~nm}$ was measured with a microplate reader (BioTek, Winooski, VT, USA). The results represent the average of three parallel samples.

Colony formation assay. NOZ and EHGB-1 cells were liquated as single cell suspensions and 600 cells were seeded into each well of 6-well plates. Cells were treated with shikonin $(0,0.1$, 0.2 and $0.3 \mu \mathrm{mol} / 1$ for NOZ and EHGB-1), and cultured for $\sim 14$ days. The cells were then fixed with $4 \%$ paraformaldehyde for $15 \mathrm{~min}$, and stained with $0.1 \%$ crystal violet (Sigma-Aldrich, St. Louis, MO, USA) for $30 \mathrm{~min}$. The total number of colonies ( $>50$ cells/colony) was manually counted.

Cell apoptosis assay. Cells were seeded in 6-well plates and treated with shikonin $(0,1,2$ and $3 \mu \mathrm{mol} / \mathrm{l})$ for $48 \mathrm{~h}$. After adherence, cells were harvested and washed twice with cold phosphate-buffered saline (PBS), then resuspended at a density of $1 \times 10^{6}$ cells $/ \mathrm{ml}$. Next, $300 \mu 1$ of binding buffer containing $5 \mu \mathrm{l}$ of Annexin V-FITC and $5 \mu \mathrm{l}$ of PI $(100 \mu \mathrm{g} / \mathrm{ml})$ was added to the cells, followed by incubation in the dark for $30 \mathrm{~min}$. The samples were determined using flow cytometry (BD Biosciences, San Diego, CA, USA).

Cell cycle analysis. NOZ and EHGB-1 cells were treated with shikonin in different concentrations $(0,1,2$ and $3 \mu \mathrm{mol} / \mathrm{l})$ for 48 h. Cells were harvested, washed with cold PBS, and fixed in $70 \%$ ethanol overnight. Then, the cells were washed, added with RNase and PI (Sigma-Aldrich), and incubated in the dark for $30 \mathrm{~min}$. The cells were detected by flow cytometry (BD Biosciences). The percentages of cells in each phase of the cell cycle were analyzed by CellQuest acquisition software (BD Biosciences).

Hoechst 33342 staining. After treatment with shikonin ( 0,1 , 2 and $3 \mu \mathrm{mol} / \mathrm{l}$ ) for $48 \mathrm{~h}$, the NOZ and EHGB-1 cells were washed in cold PBS and added with methanol:acetic acid (3:1) for $10 \mathrm{~min}$. The cells were stained with Hoechst $333425 \mu \mathrm{g} / \mathrm{ml}$ for $10 \mathrm{~min}$ at $37^{\circ} \mathrm{C}$ and subsequently observed with a fluorescence microscope (Leica Biosystems, Wetzlar, Germany).

Western blot analysis. Cells were treated with shikonin (0, $1,2$ and $3 \mu \mathrm{mol} / \mathrm{l})$ for $48 \mathrm{~h}$, and then harvested, washed and lysed in RIPA buffer (Beyotime Institute of Biotechnology, Beijing, China) and protease inhibitor (Roche Applied Science, Indianapolis, IN, USA) at $4^{\circ} \mathrm{C}$ for $5 \mathrm{~min}$. The lysates were centrifugated at $14,000 \times \mathrm{g}$ for $5 \mathrm{~min}$, the supernatant

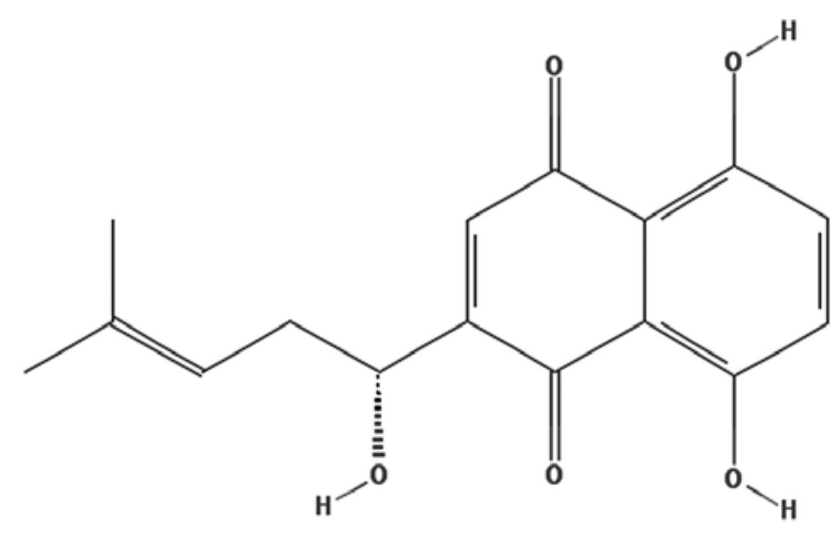

Figure 1. Chemical structure of shikonin.

was collected, and the protein concentration was detected by a bicinchoninic acid assay kit (Beyotime Institute of Biotechnology). The proteins were loaded on a $10 \%$ sodium dodecyl sulfate-polyacrylamide gel electrophoresis and transferred to nitrocellulose membranes (Millipore, Bedford, MA, USA). The membranes were blocked with 5\% skim milk, and incubated with primary antibodies against Bcl-2, Bax, cleaved caspase-3, cleaved caspase-9, cleaved PARP, cyclin D1, CDK4 and GAPDH overnight. The membrane was washed with Trisbuffered saline with Tween-20 (TBST), and then incubated with HRP-conjugated goat anti-rabbit secondary antibodies (Abcam, Cambridge, UK) for $1.5 \mathrm{~h}$. The bands were detected with Gel Doc 2000 (Bio-Rad, Hercules, CA, USA).

In vivo tumor xenograft study. Four-week-old male athymic nude mice were purchased from the Shanghai SLAC Laboratory Animal Co., Ltd. (Shanghai, China). All procedures were approved by the Institutional Animal Care and Use Committee of Shanghai Jiao Tong University. NOZ cells were resuspended in PBS $\left(1 \times 10^{6}\right.$ cells in $\left.0.2 \mathrm{ml}\right)$, and injected into the right flank of each mouse. Twenty-four hours after inoculation, the mice were randomly divided into three groups (5 mice/group). The control group was injected with vehicle (10\% DMSO and 90\% PBS) and the others were injected with shikonin ( 1 or $3 \mathrm{mg} / \mathrm{kg}$ ) every 2 days for up to 14 days. The body weight was measured every 2 days. On day 15 , the tumor tissues were removed and weighed after sacrifice of the mice injected with lethal dose of pentobarbital. The tumor volume $(\mathrm{V})=1 / 2 \times$ length $\mathrm{x}$ width $^{2}$.

Statistical analysis. All assays were performed three dependent times, and data are expressed as means \pm SD. The Student's t-test in GraphPad Prism was applied to assess the difference between two groups (GraphPad Software, San Diego, CA, USA). $\mathrm{P}<0.05\left({ }^{*} \mathrm{P}<0.05,{ }^{* *} \mathrm{P}<0.01,{ }^{* * * *} \mathrm{P}<0.001\right)$ was considered to indicate a statistically significant result.

\section{Results}

Shikonin suppresses proliferation and colony forming of GBC cells. The cell proliferation was assessed by MTT assay. We discovered an obvious reduction in the viability of shikonin-treated cells (Fig. 2A). The inhibitory concentration 
A

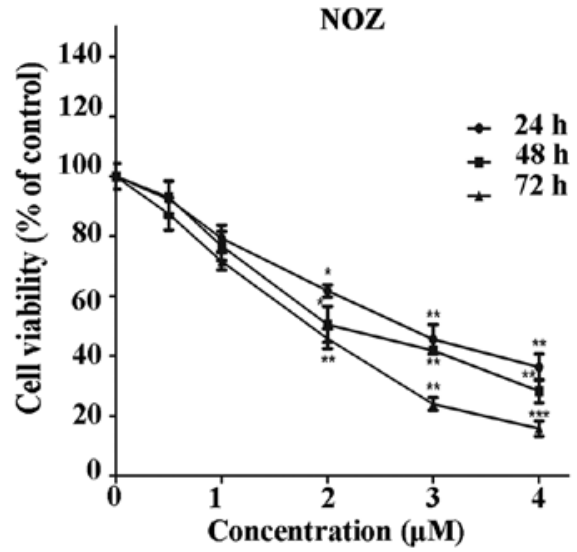

B

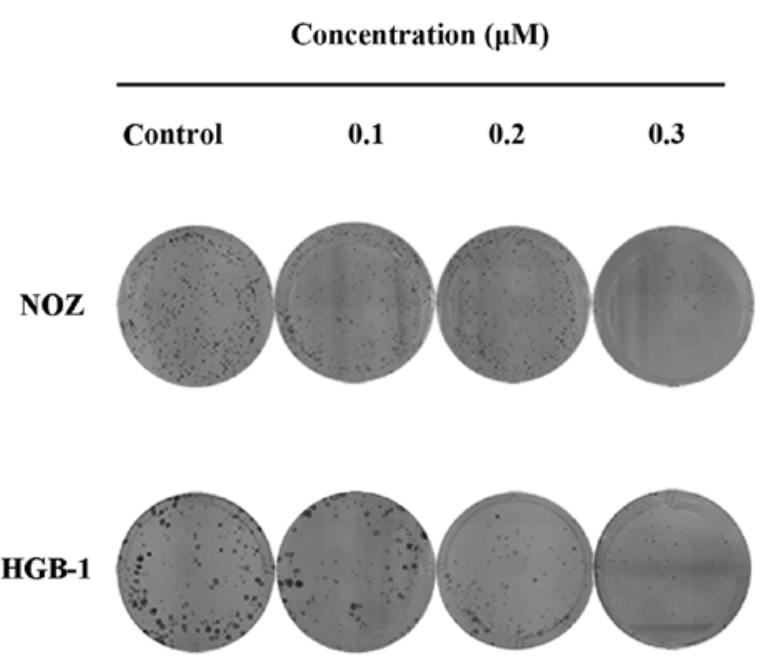

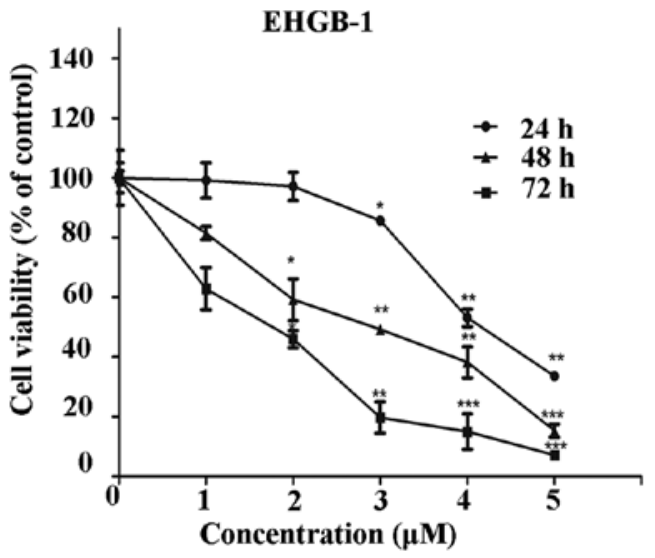

C
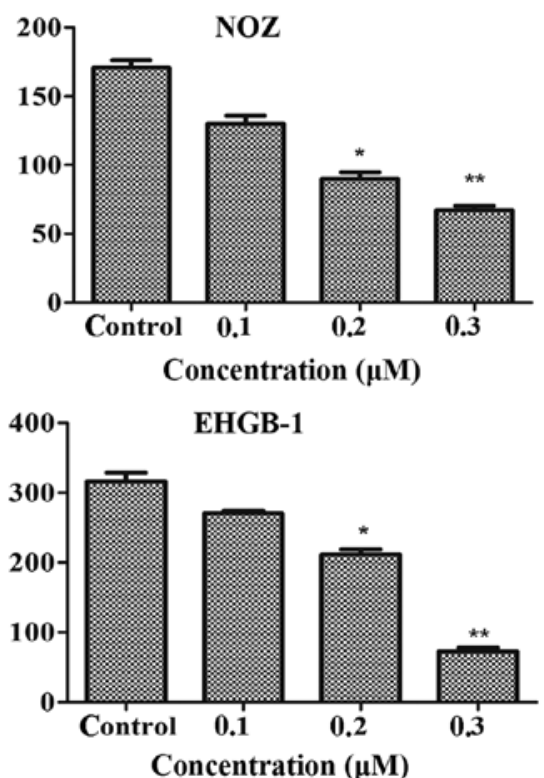

Figure 2. (A) Shikonin suppresses proliferation and colony forming of GBC cells. Cell viability was determined separately by MTT assay following treatment with shikonin for 24, 48 and $72 \mathrm{~h}$. (B) NOZ and EHGB-1 cells were treated with different concentrations of shikonin (0, $0.1,0.2$ and $0.3 \mu$ mol/1) for 14 days. (C) The colony counts are shown. All data are expressed as means $\pm \mathrm{SD}$, obtained from three independent experiments; ${ }^{*} \mathrm{P}<0.05,{ }^{* *} \mathrm{P}<0.01,{ }^{* * *} \mathrm{P}<0.001$.

(IC $)_{50}$ for NOZ and EHGB-1 cells was $\sim 2 \mu \mathrm{mol} / 1$ at $48 \mathrm{~h}$ treatment. The ability of NOZ and EHGB-1 cells to form colonies when treated with shikonin was demonstrated by the colony formation assay (Fig. 2B). The result suggested that shikonin exerted a negative influence on colony formation ability. Furthermore, we found less amount of clone formations in shikonin-treated groups than those in the control groups (Fig. 2C). These results indicated that shikonin obviously suppressed the proliferation and colony forming of NOZ and EHGB-1 cells.

Shikonin induces mitochondrial-dependent apoptosis via the $J N K$ signaling pathway in GBC cells. The impacts of shikonin on apoptosis in the NOZ and EHGB-1 cells were detected using Annexin V/PI staining and flow cytometry. As shown in Fig. 3A, the percentage of surviving cells was reduced, whereas the percentage of apoptotic cells was obviously increased (Fig. 3B).

To further confirm the cell apoptosis, we applied Hoechst 33342 staining to examine the change in nuclear morphology. The untreated cells were round with uniformity in chromatin distribution, while vivid chromatin condensation and lobulated nuclear fragmentation were revealed in cells treated with shikonin (Fig. 3C). Furthermore, the proportion of apoptotic nuclei that was obviously increased agreed with the gradually increased shikonin concentration.

Proteins of the Bcl-2 and caspase family play major roles in initiation and maintenance of mitochondrial apoptosis (19). To further explore the molecular mechanism underlying shikonin-mediated apoptosis, we evaluated the quantitative levels of apoptosis-related proteins via western blot analysis, including Bax, bcl-2, cleaved caspase-9, cleaved caspase-3 and cleaved PARP. As shown in Fig. 4A, we observed increased expression of Bax, cleaved caspase-9, cleaved caspase- 3 and cleaved PARP and downregulation of Bcl-2. Moreover, the Bcl-2/Bax ratio represents apoptotic activity (20), as cell apoptosis is promoted when the ratio decreases. In the present study, we found a significant decrease in the ratio of $\mathrm{Bcl}-2 /$ Bax in the shikonin-treated cells (Fig. 4B). We also observed 
A

NOZ

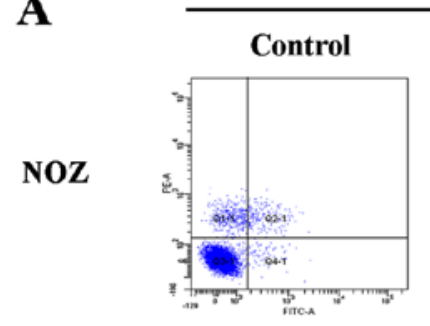

Shikonin ( $\mu \mathbf{M})$

1
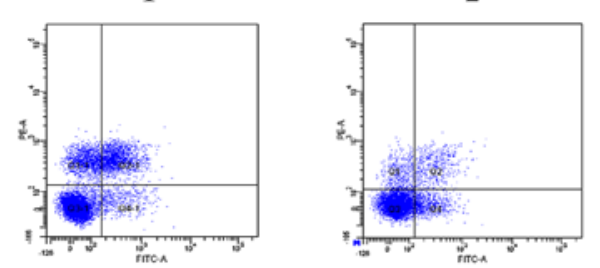

3
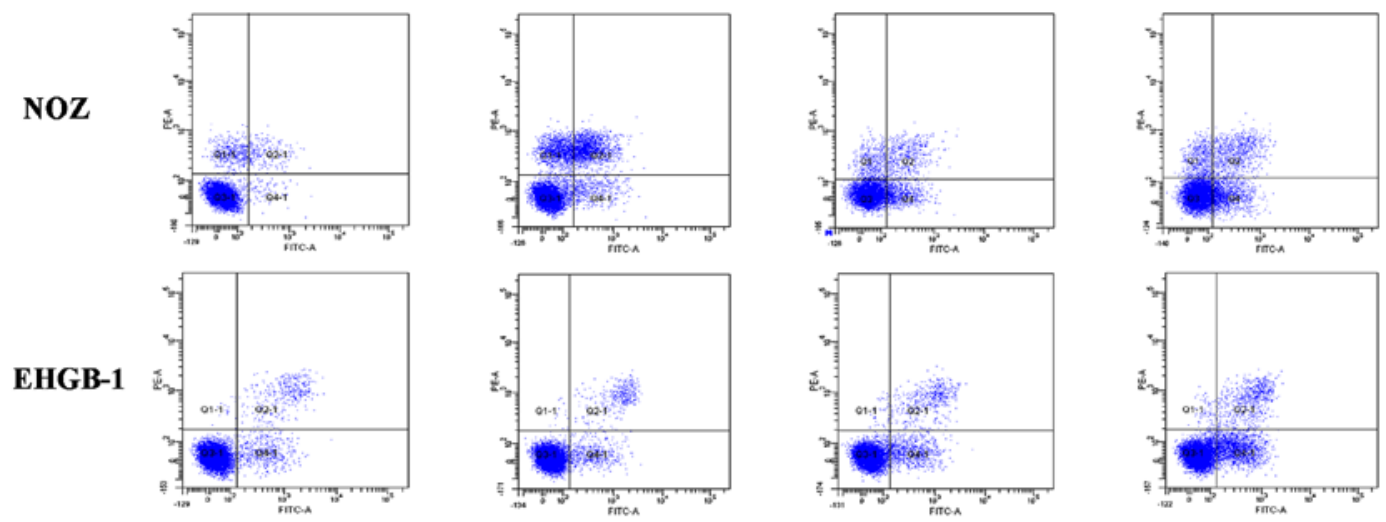

B
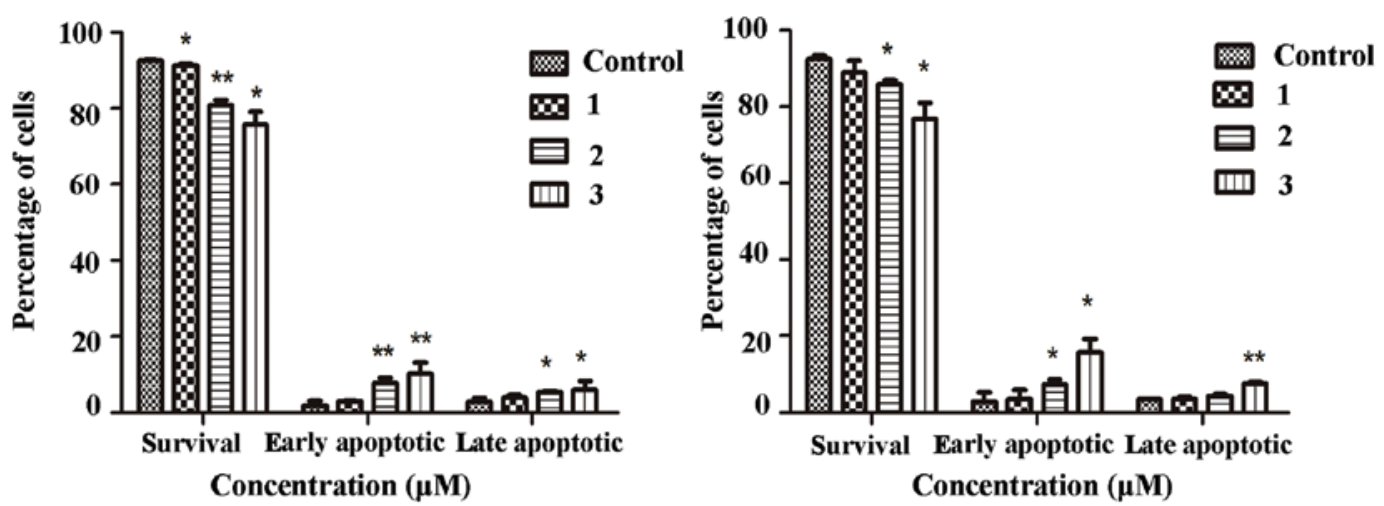

C
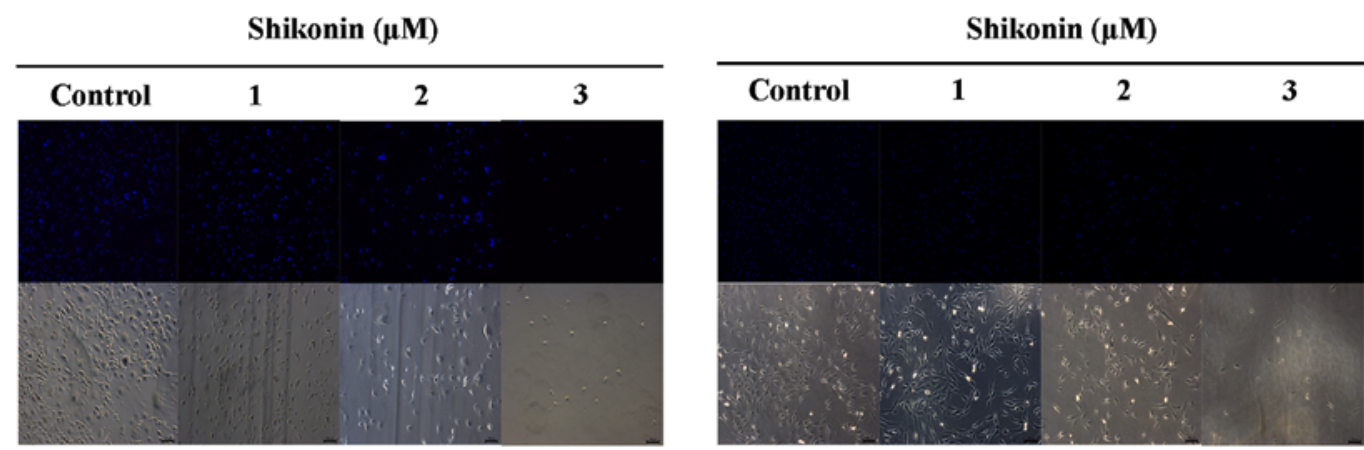

NOZ

EHGB-1

Figure 3. Shikonin induces apoptosis in GBC cells. (A) NOZ and EHGB-1 cells were stained with Annexin V-FITC/PI and analyzed by flow cytometry following treatment with shikonin $(0,1,2$ and $3 \mu \mathrm{mol} / \mathrm{l})$ for $48 \mathrm{~h}$. The right lower quadrant and right upper quadrant represent the percentage of early and late apoptotic cells, respectively. (B) The proportion of apoptotic cells is shown (mean \pm SD). (C) Differences in nuclear morphology were determined by Hoechst 33342 staining and observed under fluorescence microscopy. Representative images from three independent experiments are shown; ${ }^{*} \mathrm{P}<0.05,{ }^{* *} \mathrm{P}<0.01$ vs. the control group.

upregulation of p-JNK while no significant change in the expression of JNK was noted.

Together, these results revealed that shikonin may initiate mitochondrial-dependent apoptosis via the JNK signaling pathway in GBC cells.

Shikonin triggers $G 0 / G 1$ phase arrest by regulating cell cycle-related proteins in GBC cells. The percentage of cells in each phase of the cell cycle was determined via flow cytometry. We found an obvious increase in the proportion of $\mathrm{G} 0$ / G1 cells (Fig. 5B), indicating that shikonin suppressed cell cycle progression in the NOZ and EHGB-1 cells (Fig. 5A). We detected the expression of cell cycle-related proteins cyclin D1 and CDK4, and observed an obvious decrease in the NOZ and EHGB-1 cells (Fig. 5C). Therefore, shikonin may inhibit GBC cell proliferation by triggering G0/G1 phase arrest.

Shikonin exhibits anticancer effects in vivo. To further detect whether shikonin suppresses tumor growth in vivo, nude mice with palpable tumor xenografts were injected with vehicle (10\% DMSO and 90\% PBS) or shikonin (1 and $3 \mathrm{mg} / \mathrm{kg}$ ) every other day. This dose was determined to be effective $(21,22)$ and 
A

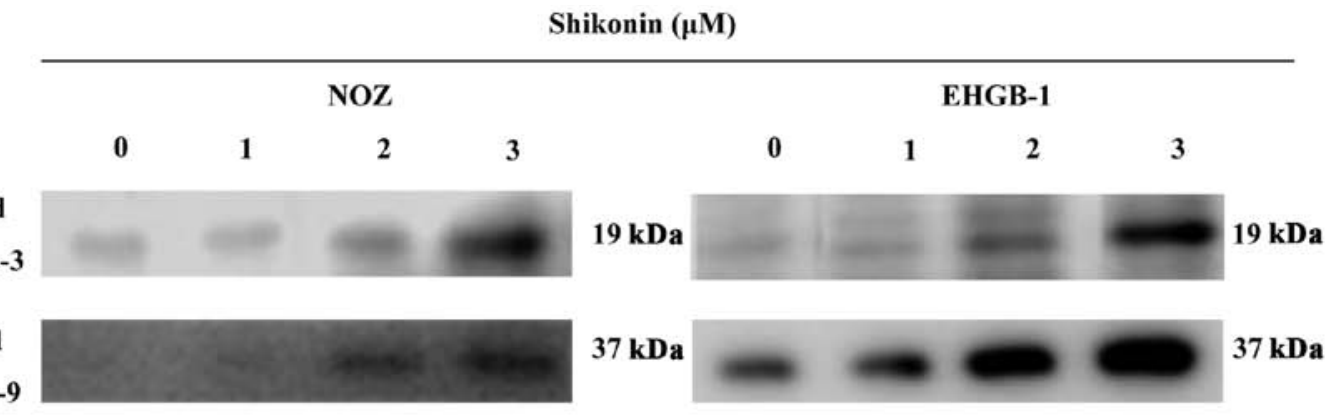

Bcl-2
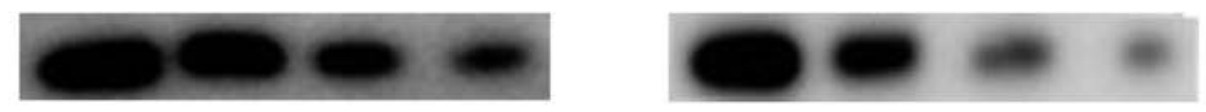

Bax
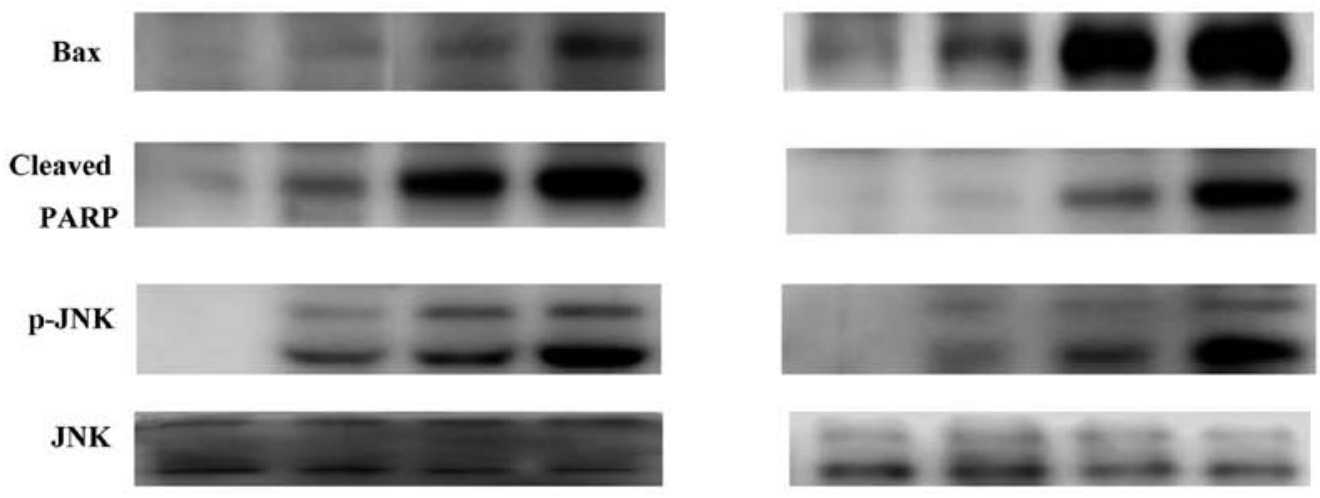

GAPDH
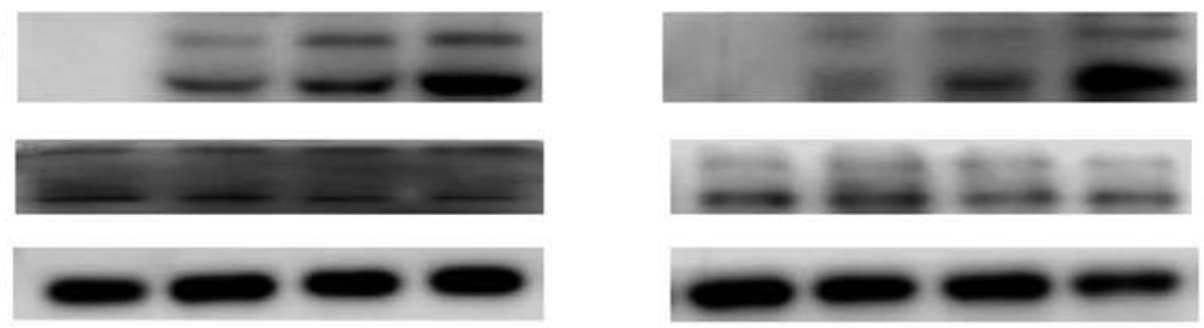

B
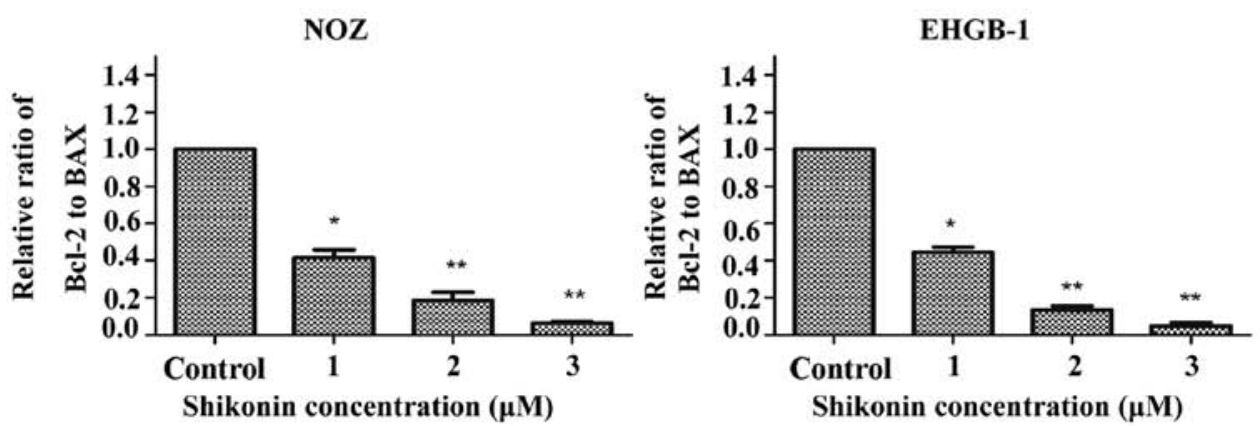

Figure 4. Shikonin induces apoptosis via the JNK signaling pathway in GBC cells. (A) NOZ and EHGB-1 cells were treated with shikonin (0, 1, 2, and $3 \mu \mathrm{mol} / \mathrm{l})$ for $48 \mathrm{~h}$. The expression levels of Bcl-2, Bax, cleaved caspase-9, cleaved caspase-3, cleaved PARP, p-JNK and JNK were determined by western blot analysis. (B) Relative ratio of Bcl-2/Bax are shown; ${ }^{*} \mathrm{P}<0.05,{ }^{* *} \mathrm{P}<0.01$ vs. the control group.

a non-toxic level; $\mathrm{LD}_{50}$ of shikonin in the mouse is $20 \mathrm{mg} / \mathrm{kg}$ when injected in an intraperitoneal manner (23). After injection, the bile and liver were previously found to contain the highest levels of shikonin, and most of the excreted metabolite was transformed (24). As shown in Fig. 6A-D, the shikonin group produced the smaller tumors when compared with the tumor volume of the vehicle group. The results demonstrated that tumor growth was obviously suppressed in the shikonintreated mice in a dose-dependent manner. Moreover, we found no significant difference in regards to body weight between the vehicle and shikonin-treated groups (Fig. 6E), suggesting that shikonin had no side-effects in nude mice. As shown in Fig. 6F, we detected the upregulation of Bax, cleaved caspase-9, cleaved caspase- 3 and cleaved PARP and downregulation of $\mathrm{Bcl}-2$ in the tumor tissues by western blot assay.

\section{Discussion}

Recently, various studies have shown that shikonin acts as an anticancer agent. However, the effects of shikonin on gallbladder cancer (GBC) cells required elucidation. In the present study, we demonstrated that shikonin induced apoptosis and G0/G1 phase arrest in human GBC cells.

The cytotoxic effects of shikonin were assessed by MTT and colony formation assays. We found that shikonin significantly inhibited cell growth in a time- and dose-dependent manner. To better understand the apoptotic effect of shikonin in GBC cells, we performed flow cytometric analysis and Hoechst 33342 staining. The results showed that shikonin induced apoptosis in GBC cells. To date, two pathways play crucial roles in cell apoptosis, including the mitochondrial intrinsic pathway 
A

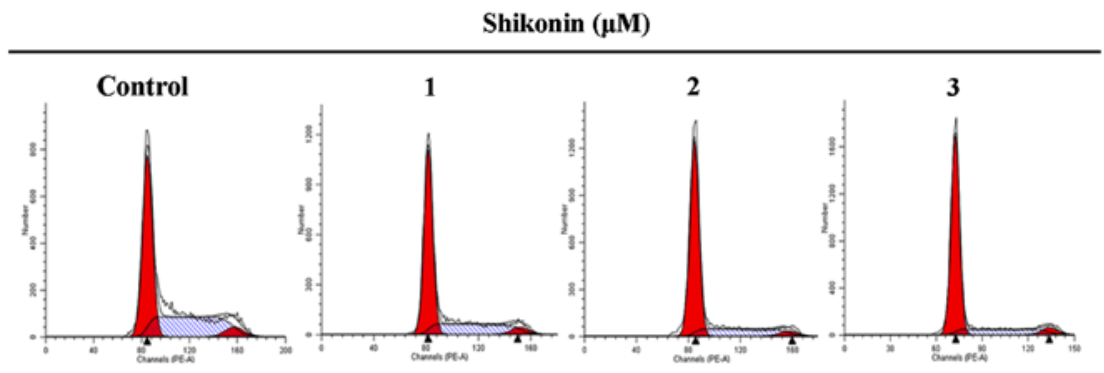

EHGB-1

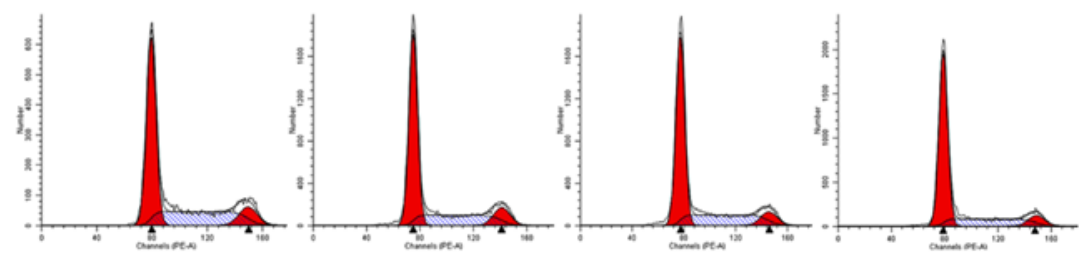

B
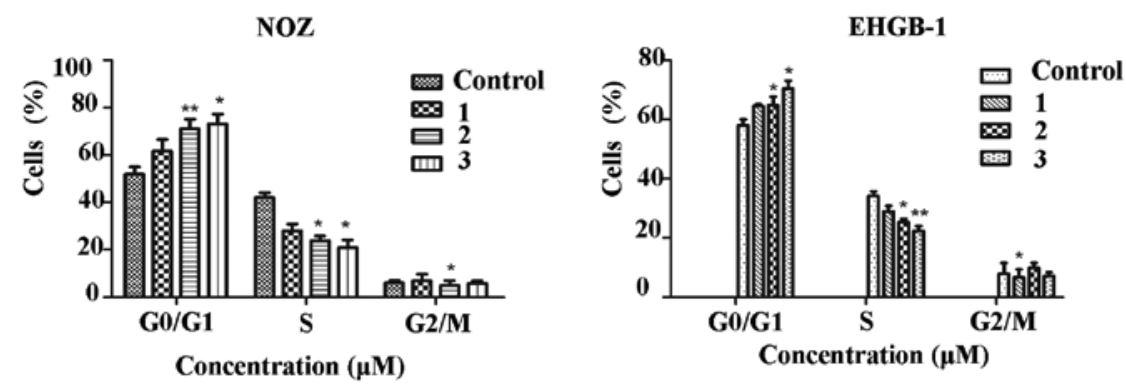

C

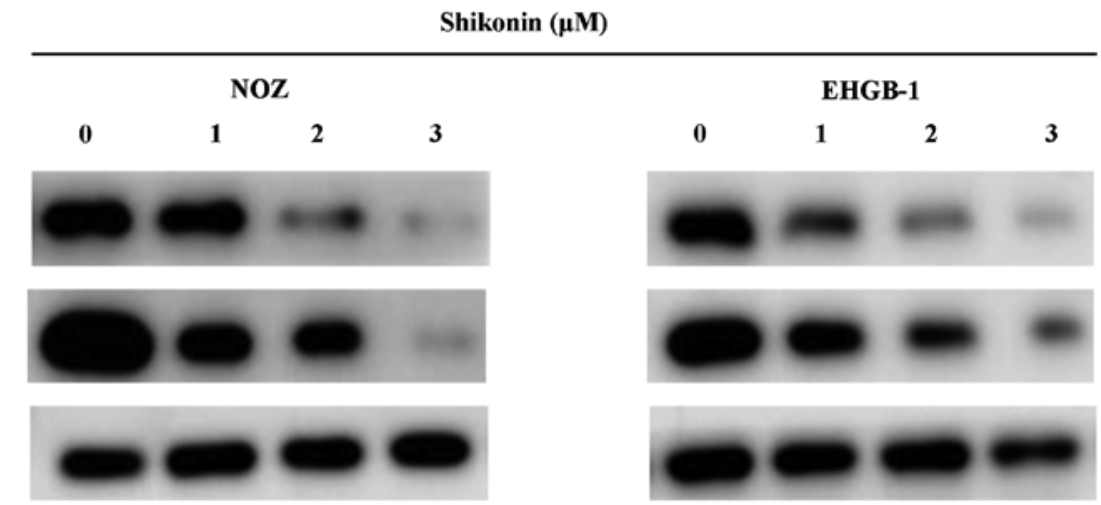

Figure 5. Shikonin triggers G0/G1 phase arrest via regulating cell cycle-related proteins in GBC cells. (A) The percentages of cells in each cell cycle were determined by flow cytometry. (B) Data are presented as the mean \pm SD $(n=3)$. (C) Western blot analysis of expression levels of cyclin D1 and CDK4. Representative blots from three independent experiments are shown; ${ }^{*} \mathrm{P}<0.05,{ }^{* *} \mathrm{P}<0.01$ vs. the control group.

and the death receptor-induced extrinsic pathway (25). In the present study, we demonstrated the importance of the intrinsic pathway in shikonin-induced apoptosis. Proteins in the Bcl-2 family are crucial regulators of the mitochondrial-dependent apoptotic pathway (26). Increased expression levels of Bax and reduced expression of Bcl-2, the dominant inhibitor of Bax, were observed in the present study. Moreover, the ratio of $\mathrm{Bcl}-2 / \mathrm{Bax}$ is a critical factor which determines the apoptosis threshold (27). In the present study, we detected that the Bcl-2/Bax ratio was significantly decreased following treatment with shikonin. These results suggest that shikonin induced apoptosis in the GBC cells.

We also detected the expression level of caspase and cleaved PARP. Caspase-9 is activated in a mitochondrial-dependent intrinsic pathway, which then mediates the activation of caspase-3 (28). Caspase-3 is an important molecule to trigger the cleavage of downstream proteins, such as PARP, in the caspase-dependent apoptosis pathway, eventually leading to apoptosis (29). PARP can be triggered in cells undergoing stress stimulus, resulting in chromatin lysis and finally triggering apoptosis (30). In the present study, we observed an obvious upregulation of cleaved caspase-3, and -9, and PARP.

Shikonin has been shown to induce cell apoptosis and inhibit proliferation in dozens of cancers through various signaling pathways, such as Erk (31), PI3K/AKT (32) and $\mathrm{NF}-\kappa \mathrm{B}$ signaling pathways (33). In the present study, we detected the expression levels of JNK and p-JNK. JNK plays an essential role in the intrinsic apoptotic pathway (34). After initially activated by extracellular stimuli, JNK activates caspase-9 and inhibits the anti-apoptotic protein Bcl-2 (35). 


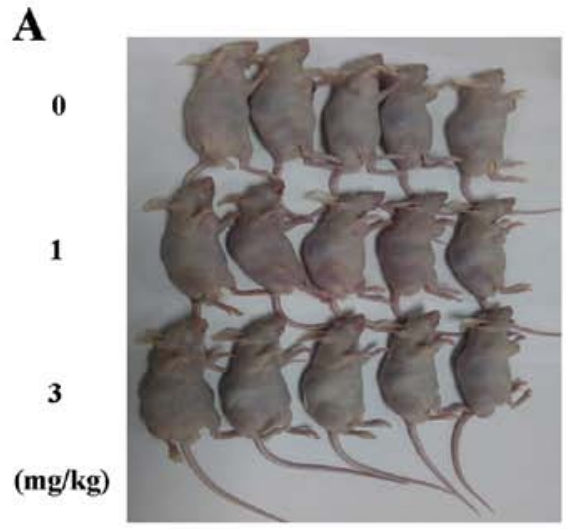

C

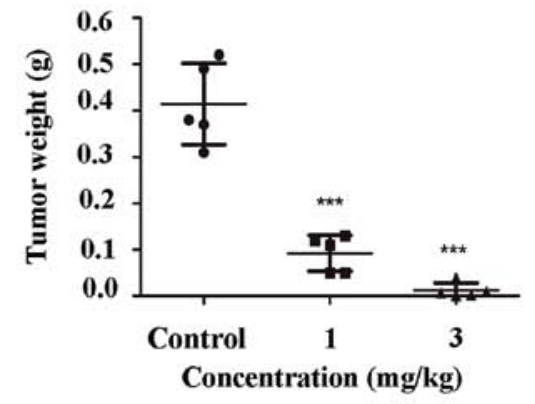

$\mathbf{E}$

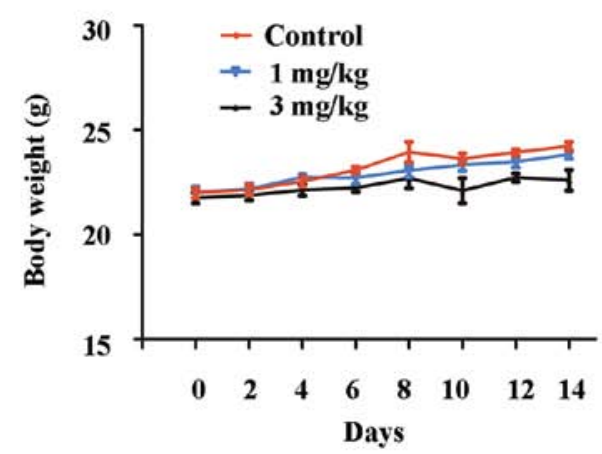

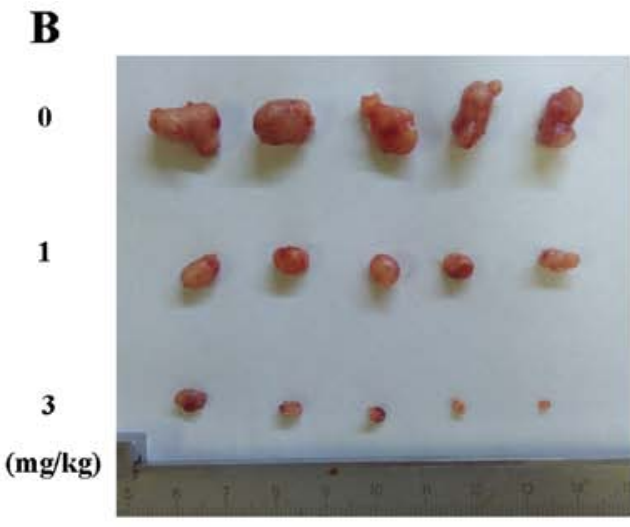

D

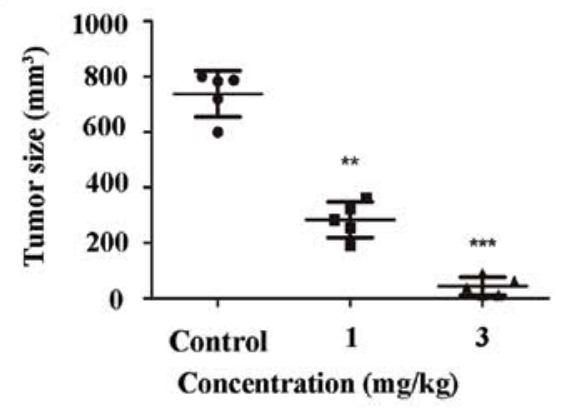

$\mathbf{F}$

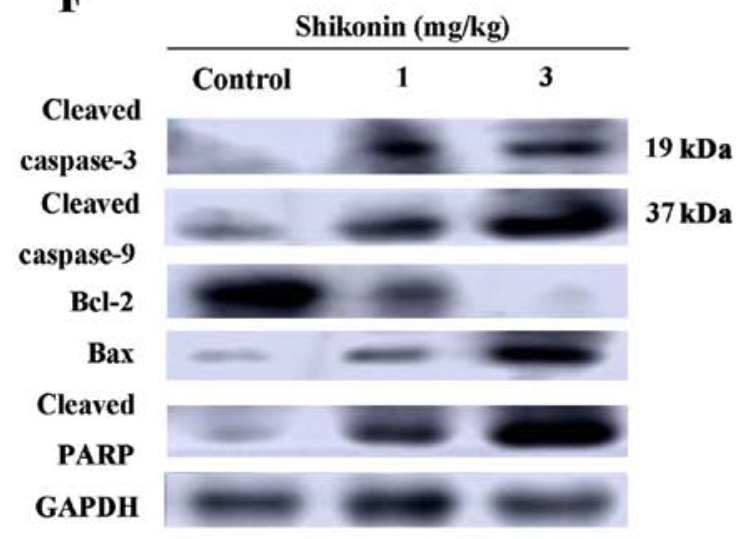

Figure 6. Shikonin exhibits anticancer effect in vivo. (A) Nude mice were injected with NOZ cells to establish tumor xenografts. Subsequently, the mice were injected with a vehicle (10\% dimethyl sulfoxide and $90 \%$ phosphate-buffered saline) or shikonin (1 and $3 \mathrm{mg} / \mathrm{kg}$ ) for up to 14 days. The sizes of tumors of the mice are shown in the images. (B-D) Tumors were collected after sacrificing the animals and subjected to weighing on day 14. (E) The body weight of the nude mice was measured during the administration period $(\mathrm{n}=5) ;{ }^{* *} \mathrm{P}<0.01,{ }^{* * *} \mathrm{P}<0.001 \mathrm{vs}$. the control group. (F) The expression levels of apoptosis-related molecules in the tumors were detected by western blot analysis.

The data revealed upregulation of $\mathrm{p}-\mathrm{JNK}$ and no obvious change in JNK, suggesting that JNK was activated in the shikonin-treated GBC cells. All in all, shikonin may trigger the apoptosis of GBC cells via the JNK signaling pathway, subsequently enhancing cell death.

Blockage of cell cycle progression has been considered as an effective strategy for the treatment of human malignancies (33). In the present study, cell cycle analysis showed that the G0/G1 phase arrest of GBC cells was induced by shikonin. The G0/ G1 phase of the cell cycle is regulated by cell cycle checkpoint proteins, such as cycling D1 and CDK4 (36), which was verified by the downregulation of CDK4 and cyclin D1 by western blot analysis.
To further confirm the apoptotic effects of shikonin, we established xenograft tumors in nude mice, and then treated them with shikonin. According to the difference in tumor volume, weight and apoptosis-related molecules in the tumors removed from the sacrificed mice, the antitumor effect of shikonin in vivo was verified. The insignificant change in body weight among the three groups indicated the safety of shikonin in the treatment of GBC.

In conclusion, the results demonstrated that shikonin exhibited marked anticancer effects by inducing apoptosis via the JNK signaling pathway and G0/G1 phase arrest. Moreover, tumor growth was also inhibited in vivo with no side-effects. Therefore, shikonin may be a novel and safe chemotherapeutic agent for the treatment of GBC. 


\section{Acknowledgements}

The present study was supported by the National Natural Science Foundation of China (no. 81372642).

\section{References}

1. Li Z, Yu X, Shen J, Law PT, Chan MT and Wu WK: MicroRNA expression and its implications for diagnosis and therapy of gallbladder cancer. Oncotarget 6: 13914-13921, 2015.

2. Subbannayya T, Leal-Rojas P, Barbhuiya MA, Raja R, Renuse S, Sathe G, Pinto SM, Syed N, Nanjappa V, Patil AH, et al: Macrophage migration inhibitory factor - a therapeutic target in gallbladder cancer. BMC Cancer 15: 843, 2015.

3. Shu YJ, Bao RF, Jiang L, Wang Z, Wang XA, Zhang F, Liang HB, Li HF, Ye YY, Xiang SS, et al: MicroRNA-29c-5p suppresses gallbladder carcinoma progression by directly targeting CPEB4 and inhibiting the MAPK pathway. Cell Death Differ 24: 445-457, 2017.

4. Shu YJ, Weng H, Ye YY, Hu YP, Bao RF, Cao Y, Wang XA, Zhang F, Xiang SS, Li HF, et al: SPOCK1 as a potential cancer prognostic marker promotes the proliferation and metastasis of gallbladder cancer cells by activating the PI3K/AKT pathway. Mol Cancer 14: 12, 2015.

5. Nagaraja V and Eslick GD: Systematic review with meta-analysis: The relationship between chronic Salmonella typhi carrier status and gall-bladder cancer. Aliment Pharmacol Ther 39: 745-750, 2014.

6. Carriaga MT and Henson DE: Liver, gallbladder, extrahepatic bile ducts, and pancreas. Cancer 75 (Suppl 1): S171-S190, 1995

7. Jung W, Jang JY, Kang MJ, Chang YR, Shin YC, Chang J and Kim SW: Effects of surgical methods and tumor location on survival and recurrence patterns after curative resection in patients with T2 gallbladder cancer. Gut Liver 10: 140-146, 2016

8. Li M, Zhang Z, Li X, Ye J, Wu X, Tan Z, Liu C, Shen B, Wang XA $\mathrm{Wu} \mathrm{W}$, et al: Whole-exome and targeted gene sequencing of gallbladder carcinoma identifies recurrent mutations in the ErbB pathway. Nat Genet 46: 872-876, 2014.

9. Zhao S, Cao Y, Liu SB, Wang XA, Bao RF, Shu YJ, Hu YP, Zhang YJ, Jiang L, Zhang F, et al: The E545K mutation of PIK3CA promotes gallbladder carcinoma progression through enhanced binding to EGFR. JJ Exp Clin Cancer Res 35: 97, 2016.

10. Marcano-Bonilla L, Mohamed EA, Mounajjed T and Roberts LR: Biliary tract cancers: Epidemiology, molecular pathogenesis and genetic risk associations. Linchuang Zhongliuxue Zazhi 5: 61, 2016.

11. Bao RF, Shu YJ, Hu YP, Wang XA, Zhang F, Liang HB, Ye YY, $\mathrm{Li} \mathrm{HF}$, Xiang SS, Weng $\mathrm{H}$, et al: miR-101 targeting ZFX suppresses tumor proliferation and metastasis by regulating the MAPK/Erk and Smad pathways in gallbladder carcinoma. Oncotarget 7: 22339-22354, 2016.

12. Yang F, Chen Y, Duan W, Zhang C, Zhu H and Ding J: SH-7, a new synthesized shikonin derivative, exerting its potent antitumor activities as a topoisomerase inhibitor. Int J Cancer 119: 1184-1193, 2006

13. Singh F, Gao D, Lebwohl MG and Wei H: Shikonin modulates cell proliferation by inhibiting epidermal growth factor receptor signaling in human epidermoid carcinoma cells. Cancer Lett 200 $115-121,2003$

14. Lu D, Qian J, Li W, Feng Q, Pan S and Zhang S: $\beta$-hydroxyisovaleryl-shikonin induces human cervical cancer cell apoptosis via PI3K/AKT/mTOR signaling. Oncol Lett 10: 3434-3442, 2015.

15. Liu JP, Liu D, Gu JF, Zhu MM and Cui L: Shikonin inhibits the cell viability, adhesion, invasion and migration of the human gastric cancer cell line MGC-803 via the Toll-like receptor 2/nuclear factor-kappa B pathway. J Pharm Pharmacol 67: 1143-1155, 2015.

16. Yang H, Zhou P, Huang H, Chen D, Ma N, Cui QC, Shen S, Dong W, Zhang X, Lian W, et al: Shikonin exerts antitumor activity via proteasome inhibition and cell death induction in vitro and in vivo. Int J Cancer 124: 2450-2459, 2009.

17. Komi Y, Suzuki Y, Shimamura M, Kajimoto S, Nakajo S, Masuda M, Shibuya M, Itabe H, Shimokado K, Oettgen P, et al: Mechanism of inhibition of tumor angiogenesis by beta-hydroxyisovalerylshikonin. Cancer Sci 100: 269-277, 2009.
18. Chen J, Xie J, Jiang Z, Wang B, Wang Y and Hu X: Shikonin and its analogs inhibit cancer cell glycolysis by targeting tumor pyruvate kinase-M2. Oncogene 30: 4297-4306, 2011.

19. He G, He G, Zhou R, Pi Z, Zhu T, Jiang L and Xie Y: Enhancement of cisplatin-induced colon cancer cells apoptosis by shikonin, a natural inducer of ROS in vitro and in vivo. Biochem Biophys Res Commun 469: 1075-1082, 2016.

20. Liu T, Li R, Zhao H, Deng J, Long Y, Shuai MT, Li Q, Gu H, Chen YQ and Leng AM: eIF4E promotes tumorigenesis and modulates chemosensitivity to cisplatin in esophageal squamous cell carcinoma. Oncotarget 7: 66851-66864, 2016.

21. Jeung YJ, Kim HG, Ahn J, Lee HJ, Lee SB, Won M, Jung CR, Im JY, Kim BK, Park SK, et al: Shikonin induces apoptosis of lung cancer cells via activation of FOXO3a/EGR1/SIRT1 signaling antagonized by $\mathrm{p} 300$. Biochim Biophys Acta 1863: 2584-2593, 2016

22. Wang Y, Zhou Y, Jia G, Han B, Liu J, Teng Y, Lv J, Song Z, Li Y, $\mathrm{Ji}$ L, et al: Shikonin suppresses tumor growth and synergizes with gemcitabine in a pancreatic cancer xenograft model: Involvement of NF- $\mathrm{kB}$ signaling pathway. Biochem Pharmacol 88: 322-333, 2014.

23. Hayashi M: Pharmacological studies of Shikon and Tooki. (2) Pharmacological effects of the pigment components, Shikonin and acetylshikonin. Nihon Yakurigaku Zasshi 73: 193-203, 1977.

24. Chen X, Yang L, Oppenheim JJ and Howard MZ: Cellular pharmacology studies of shikonin derivatives. Phytother Res 16: 199-209, 2002

25. Call JA, Eckhardt SG and Camidge DR: Targeted manipulation of apoptosis in cancer treatment. Lancet Oncol 9: 1002-1011, 2008.

26. Min R, Tong J, Wenjun Y, Wenhu D, Xiaojian Z, Jiacai H, Jian Z, Wantao $C$ and Chenping Z: Growth inhibition and induction of apoptosis in human oral squamous cell carcinoma Tca-8113 cell lines by Shikonin was partly through the inactivation of NF-kappaB pathway. Phytother Res 22: 407-415, 2008.

27. Wang W, Guo Q, You Q, Zhang K, Yang Y, Yu J, Liu W, Zhao L, $\mathrm{Gu} \mathrm{H}, \mathrm{Hu} \mathrm{Y}$, et al: Involvement of bax/bcl-2 in wogonin-induced apoptosis of human hepatoma cell line SMMC-7721. Anticancer Drugs 17: 797-805, 2006.

28. Hill MM, Adrain C and Martin SJ: Portrait of a killer: The mitochondrial apoptosome emerges from the shadows. Mol Interv 3: 19-26, 2003.

29. Nuñez G, Benedict MA, Hu Y and Inohara N: Caspases: The proteases of the apoptotic pathway. Oncogene 17: 3237-3245, 1998.

30. Wang Y, An R, Umanah GK, Park H, Nambiar K, Eacker SM, Kim B, Bao L, Harraz MM, Chang C, et al: A nuclease that mediates cell death induced by DNA damage and poly(ADPribose) polymerase-1. Science 354: 354, 2016

31. Jing H, Sun W, Fan J, Zhang Y, Yang J, Jia J, Li J, Guo J, Luo S and Zheng Y: Shikonin induces apoptosis of HaCaT cells via the mitochondrial, Erk and Akt pathways. Mol Med Rep 13: 3009-3016, 2016

32. Zhang FY, Hu Y, Que ZY, Wang P, Liu YH, Wang ZH and Xue YX: Shikonin inhibits the migration and invasion of human glioblastoma cells by targeting phosphorylated $\beta$-catenin and phosphorylated PI3K/Akt: A potential mechanism for the anti-glioma efficacy of a Traditional Chinese Herbal Medicine. Int J Mol Sci 16: 23823-23848, 2015

33. Tian R, Li Y and Gao M: Shikonin causes cell-cycle arrest and induces apoptosis by regulating the EGFR-NF- $\kappa \mathrm{B}$ signalling pathway in human epidermoid carcinoma A431 cells. Biosci Rep 35: 35, 2015

34. Schroeter H, Boyd CS, Ahmed R, Spencer JP, Duncan RF, Rice-Evans C and Cadenas E: c-Jun N-terminal kinase (JNK)mediated modulation of brain mitochondria function: New target proteins for JNK signalling in mitochondrion-dependent apoptosis. Biochem J 372: 359-369, 2003.

35. Nakamura Y: Chemoprevention by isothiocyanates: Molecular basis of apoptosis induction. Forum Nutr 61: 170-181, 2009.

36. Nakanishi M, Shimada $M$ and Niida $H$ : Genetic instability in cancer cells by impaired cell cycle checkpoints. Cancer Sci 97: 984-989, 2006 